\title{
EEES y la formación en comunicación en España. La estructura de la oferta de posgrado
}

\author{
Antonio CAstillo EsParcia \\ Universidad de Málaga \\ acastilloe@uma.es \\ Alejandro Álvarez NoBell \\ Universidad San Jorge (Zaragoza) \\ aalvarez@usj.es \\ José Antonio MuÑIZ VeLÁZQUEZ \\ Universidad San Jorge (Zaragoza) \\ jamuniz@usj.es
}

Recibido: $14 / 11 / 2012$

Aceptado: 23/01/2013

\section{Resumen}

En la actual sociedad del conocimiento y de la información, la comunicación se ha convertido en un elemento vertebrador de numerosos aspectos sociales, económicos y políticos que implica la necesidad de que empresas, entidades sociales e instituciones fomenten la I+D + I. Las universidades, enmarcadas en el EEES, deben protagonizar este proceso con sus políticas de investigación y formación. En España, las recientes modificaciones normativas sobre posgrado (RD 99/2011 y RD 1393/2007) han configurado un nuevo mapa de titulaciones que hace necesario conocer su nivel de implantación y características. Este texto persigue reflexionar sobre la estructura de la oferta en España de programas de posgrado (Máster oficiales y Doctorado) en comunicación existentes en 40 universidades (públicas y privadas), sus objetivos formativos (investigador y/o profesional), distribución geográfica, plazas y demás características configurativas.

Palabras clave: EEES, España, Comunicación, Posgrado, Doctorado, Máster

\section{EHEA and education in communication in Spain. The supply structure of postgraduate}

\begin{abstract}
Communication has become a backbone of many social, economic and political issues in the current information and knowledge society, leading companies, social organizations, and institutions to make an additional effort on $\mathrm{R}+\mathrm{D}+\mathrm{I}$. Universities, in the framework of the European Higher Education Area, are ideally placed to lead this process with their research and training policies. In Spain, recent regulatory changes on graduate study levels (RD 99/2011 and RD 1393/2007) have resulted in a new map of university degrees with varying levels of implementation and different features. This text aims to reflect on the supply structure, the training goals (research and/or professional), the geographic distribution, the available places, and other defining features of Spanish postgraduate programs (official Master and Doctoral) in Communication offered by 40 universities (public and private).

Keywords: EHEA, Spain, Communication, Postgraduate, Doctoral, Master.

\section{Referencia normalizada}

CASTILLO ESPARCIA, Antonio; ÁLVAREZ NOBELL, Alejandro; y MUÑIZ VELÁZQUEZ, José Antonio (2013): "EEES y la formación en comunicación en España. La estructura de la oferta de posgrado". Estudios sobre el Mensaje Periodístico. Vol. 19, Núm. especial marzo, págs.: 99-110. Madrid, Servicio de Publicaciones de la Universidad Complutense.
\end{abstract}

Sumario: 1. Introducción. 1.1. Los estudios universitarios oficiales de Posgrado. 1.2. Los posgrados en comunicación de España. 2. Metodología. 3. Desarrollo. 3.1. Tipo de Universidad. 3.2. Áreas temáticas 
de los másteres. 3.3. La oferta de posgrado por Universidad. 3.4. Distribución territorial según el tipo de Universidad. 3.5. Orientación de las titulaciones. 4. Conclusiones. 5. Referencias bibliográficas.

\section{Introducción}

En la actual sociedad del conocimiento y de la información, la comunicación se ha convertido en un elemento vertebrador de numerosos aspectos sociales, económicos y políticos que implica la necesidad de que empresas, entidades sociales e instituciones desarrollen una labor de fomento de la Investigación, en Desarrollo y la Innovación $(\mathrm{I}+\mathrm{D}+\mathrm{I})$. El modelo productivo de la economía necesita a los investigadores como actores principales de la sociedad en la generación, transferencia y adecuación de la $\mathrm{I}+\mathrm{D}+\mathrm{I}$. Los investigadores han de jugar un papel esencial en todas las instituciones implicadas en la innovación y la investigación, de forma que lideren el trasvase desde el conocimiento hasta el bienestar de la sociedad.

Este proceso se viene desarrollando en un marco que tuvo comienzo en mayo de 1998, cuando los ministros encargados de la educación superior de Alemania, Francia, Italia y el Reino Unido suscribieron en París la Declaración de la Sorbona, instando al desarrollo de un Espacio Europeo de la Educación Superior (EEES). Siguiendo el objetivo iniciado en la Sorbona, un año más tarde, los 29 países europeos, entre los que se encontraba España, celebraron una conferencia en Bolonia, que sentó las bases para conseguir un EEES en la frontera del año 2010. La declaración de Bolonia (Olmedo Salar, et al, 2011: 60) marcaba los objetivos para adoptar un sistema fácilmente legible y comparable de titulaciones basado en dos niveles principales (grado y postgrado); establecer un sistema internacional de créditos; promover la movilidad de estudiantes, profesores e investigadores; y la cooperación europea para garantizar la calidad de la educación superior.

Evidentemente la Universidad española no ha sido ajena a las profundas transformaciones normativas, académicas, investigadoras y formativas que la aplicación del EEES ha motivado; debatiéndose su indeclinable papel como uno de los pilares en el desarrollo de políticas de educación, investigación y transferencia. En este sentido, la presente investigación tuvo por objetivo analizar y reflexionar sobre la estructura formativa de posgrados en comunicación (Máster oficial y doctorado) en España, que se oferta durante el curso 2012/2013 en 48 universidades (públicas y privadas), sus objetivos formativos (investigador y/o profesional), distribución geográfica, plazas y demás características configurativas.

En el caso de los másteres oficiales se han implantado con rapidez y, en ocasiones, con precipitación e improvisación. Ello ha conllevado que superen en número a los grados y desde luego a los programas de doctorado. Este dato nos ha llevado a conocer qué tipo de oferta de Máster oficial se ha generado y cuáles son sus principales características. Todo ello por la significación académica y profesional que han adquirido los másteres, ya que han devenido en la puerta de acceso a la investigación al convertirse en requisito para acceder a un programa de doctorado. Asimismo, los másteres también pueden derivar hacia la capacitación profesional, por lo que su influencia también se ha extendido al ámbito social y empresarial. 


\subsection{Los estudios universitarios oficiales de posgrado}

Tras la promulgación del Real Decreto 56/2005, de 21 de enero, por el que se regulan los estudios universitarios oficiales de posgrado, se produce una profunda transformación de este tipo de estudios (Máster y doctorado) en el panorama universitario español. Las principales características son que:

- Los estudios oficiales de posgrado (denominados universitarios, a diferencia de los títulos propios) tienen como finalidad la especialización del estudiante en su formación académica, profesional o investigadora.

- Los estudios tendrán una extensión mínima de 60 créditos y máxima de 120 , y estarán dedicados a la formación avanzada, de carácter especializado o multidisciplinar, dirigido a una especialización académica o profesional, o bien a promover la iniciación en tareas investigadoras.

El estudiante, una vez obtenido un mínimo de 60 créditos en programas oficiales de posgrado o cuando se halle en posesión del título oficial de máster, podrá solicitar su admisión en el doctorado, siempre que haya completado un mínimo de 300 créditos en el conjunto de sus estudios universitarios de grado y posgrado.

A ese decreto se le añadió el $R D$ 1393/2007, de 29 de octubre, por el que se establece la ordenación de las enseñanzas universitarias oficiales que concretaba ciertos aspectos de la normativa anterior como son:

- Los planes de estudios conducentes a la obtención de los títulos de máster universitario tendrán entre 60 y 120 créditos, que contendrá toda la formación teórica y práctica que el estudiante deba adquirir: materias obligatorias, materias optativas, seminarios, practicas externas, trabajos dirigidos, trabajo de fin de máster, actividades de evaluación, y otras que resulten necesarias según las características propias de cada título.

- Estas enseñanzas concluirán con la elaboración y defensa pública de un trabajo de fin de Máster, que tendrá entre 6 y 30 créditos.

- Tras la autorización de la Comunidad Autónoma y la verificación del plan de estudios a que se refiere el artículo anterior, el Ministerio de Educación y Ciencia elevará al Gobierno la propuesta para el establecimiento del carácter oficial del título y su inscripción en el RUCT ${ }^{1}$, cuya aprobación mediante acuerdo del Consejo de Ministros será publicada en el Boletín Oficial del Estado. La inscripción en el RUCT tendrá como efecto la consideración inicial de titulo acreditado.

Finalmente, el Real Decreto 99/2011, de 28 de enero, por el que se regulan las enseñanzas oficiales de doctorado determinó que los mismos podrán ser presentados por las Escuelas de doctorados y tendrán una actividad esencialmente de tipo investigadora. Además establece plazos máximos de duración de los programas, profundizando la distinción entre los diferentes niveles universitarios. En definitiva, los antiguos doctorados reconvertidos bajos estos criterios o los nuevos programas debe-

1 Registro de Universidades, Centros y Títulos (RUCT). https://www.educacion.gob.es /ruct/home 
rían incluir para su verificación (García Jiménez, et al., 2012a: 85): la descripción del programa y datos básicos; las competencias; el acceso y admisión de estudiantes; las actividades formativas; la organización del programa; la supervisión de tesis; el seguimiento del doctorando; los recursos humanos; los recursos materiales y de apoyo; y la revisión, mejora y resultados del programa.

Esta nueva ingeniería normativa diseñada bajo los principios del EEES, ha desencadenado una estructura del Sistema Universitario Español ${ }^{2}$ compuesto por un total de 78 universidades en el curso académico 2010-2011. De esa cifra, las universidades públicas alcanzan la cantidad de 50 universidades $(64,10 \%)$ y las privadas 28 $(35,89 \%)$. Si atendemos al número total de estudiantes matriculados, se observa que las universidades públicas reciben al $88,9 \%$ del total de estudiantes, que supera la proporción del $64 \%$ de universidades públicas existentes.

En lo referido a másteres oficiales teniendo en cuenta todos los campos de conocimiento, según cifras del curso académico 2009-2010, en este nivel de estudio nos encontramos con un total de 81.840 estudiantes $(5,25 \%$ del total). Esa cantidad supone un incremento del 64,3\% respecto del curso académico 2008-2009. Estos datos son consecuencia de la multiplicación de la oferta de máster oficial en las universidades españolas, que ha pasado de 829 en el curso académico 2006-2007 a los 2.429 del curso académico 2009-2010.

Con relación a la edad, los estudiantes matriculados son un $42 \%$ en la franja de 25 30 años, un $24,7 \%$ de menos de 25 años, un $23,8 \%$ de 31 a 40 años y un $9,5 \%$ de más de 40 años. Y sobre los graduados en los másteres, el 50,2\% tiene entre 25-30 años, el $22 \%$ de 31 a 40 años, el 17,8\% menos de 25 años y el 10\% más de 40 años. Con estas cifras, la mayor tasa de abandono se produce entre los alumnos de menos de 25 años que representan el $24,7 \%$ de los estudiantes matriculados, pero sólo se gradúan el $17,8 \%$.

Finalmente, en lo referido a movilidad, los estudiantes extranjeros matriculados en másteres oficiales suponen el $18,4 \%$ del total de matriculados, siendo mayoritarios los que proceden de América Latina y el Caribe (45,6\%), seguidos de estudiantes procedentes de la Europa de los 27 (30,7\%), Norte de África 7,4\%, resto de Europa $(6,4 \%)$, Asia y Oceanía (5,5\%), resto de África $(2,8 \%)$ y, finalmente, Estados Unidos y Canadá $(1,6 \%)$. Con estos datos, se confirma que España es un país de gran demanda de enseñanza de posgrado para los estudiantes de habla hispana.

\subsection{Los posgrados en Comunicación de España}

En julio de 2011, en la Universidad de San Pablo (Brasil) tuvo lugar el primer Congreso Mundial de Comunicación Iberoamericana, promovido por la Confederación de Asociaciones Iberoamericana de Comunicación Científica y Académica (CONFIBERCOM ${ }^{3}$. En esta importante cita, la Asociación Española de Investigación de la Comunicación (AE-IC) presentó algunas referencias sobre los posgrados específicos

2 Datos y cifras del Sistema Universitario Español, curso 2010-2011, Ministerio de Educación, 2010, Madrid.

3 Organización no lucrativa educativa fundada el 18 de abril de 2009 (Madeira, Portugal) conformada por: ABOIC (Asociación Boliviana de Investigadores de la Comunicación); AE-IC 
dentro del área de la Comunicación en España, tomadas a partir de una primera exploración (55 programas) a la información disponible en los sitios web de las universidades (García Jiménez, et al., 2012b: 264):

- Existe en la generalidad de los programas de posgrado, una vinculación fuerte con los doctorados.

- Se observan un mayor número de posgrados orientados a la investigación, si bien existen también un número relevante de programas vinculados con aspectos profesionales.

- La universidad privada tiene una mayor oferta relativa que la universidad pública.

- En cuanto a los temas, se puede destacar, por orden, las siguientes tendencias: a) el entorno audiovisual, entre sus diversas variantes, es el más abordado, con cuestiones como la televisión, el cine, guion y narrativa, producción y la conexión con los nuevos medios; $b$ ) destaca en segundo lugar los programas vinculados al periodismo, en diversos aspectos (multimedia, especializado etc.); c) el mundo digital también supone un contenido muy seguido por los programas; d) hay un número representativo de posgrados que trata el campo de la comunicación social, ya sea de forma genérica o bien de forma más específica (problemas, accesibilidad, cultura etc.); e) también es objeto de estudio la conexión entre investigación y comunicación; f) hay un conjunto representativo de programas que trabajan la comunicación corporativa; y g) se detectan otros temas que, por su naturaleza, tiene menos presencia como la moda, el diseño gráfico, la nutrición, la religión o la escritura.

Frente a estos datos preliminares y las recientes modificaciones normativas del posgrado (RD 99/2011 sobre doctorado y RD 1393/2007 sobre enseñanzas oficiales) se ha configurado un nuevo mapa de titulaciones de posgrado que hace necesario indagar respecto del grado real de implantación y sus principales características.

Así, este estudio permite presentar una radiografía del contexto de las enseñanzas de los másteres oficiales y doctorados ofertados en comunicación en España en el curso 2012/2013, tomando como principales variables de estudio: la titularidad de la universidad (pública y privada), la modalidad de la enseñanza (académica, investigadora y profesional), el número de créditos ofertados, el volumen de plazas ofertadas,

(Asociación Española de Investigación de la Comunicación); ALAIC (Asociación Latinoamericana de Investigadores de la Comunicación); AMIC (Asociación Mexicana de Investigadores de la Comunicación); AssIBERCOM (Associação Ibero Americana de Comunicação); FADECCOS (Federación Argentina de Carreras de Comunicación Social); FELAFACS (Federação Latino-americana de Faculdades de Comunicação Social); INVECOM (Asociación de Investigadores Venezolanos de la Comunicación); LUSOCOM (Federação das Associações Lusófonas de Ciências da comunicação); SEICOM (Sociedad Ecuatoriana de Estudios Interdisciplinarios de la Comunicación); SOCICOM (Federação Brasileira das Associações Científicas e Acadêmicas de Comunicação); SOPCOM (Associação Portuguesa de Ciências da Comunicação) y ULEPICC (União Latina de Economía Política da Informação, da Comunicação e da Cultura). http://www.confibercom.org 
el número de alumnos, los ámbitos temáticos en las diferentes disciplinas como son periodismo, comunicación audiovisual y publicidad y relaciones públicas.

Con ello se podrá conocer la situación de la distribución de los estudios de posgrado en las diferentes comunidades, el volumen de personas que en ellos estudian y las principales características de los estudios.

\section{Metodología}

Para la investigación se empleó un muestreo no probabilístico por propósito a partir del universo de titulaciones de "Master Universitarios" y "Doctorados" presentes en el Registro de Universidades, Centros y Títulos (RUCT) del Ministerio de Educación, Cultura y Deporte de España ${ }^{4}$.

La selección se aplicó eligiendo los títulos que se imparten en la totalidad del territorio de España y que llevan en el nombre del mismo la palabra "comunicación". Sobre un total de 253 resultados iniciales en el caso de máster y 113 en doctorado, se excluyeron aquellos correspondientes a carreras de ingeniería, telecomunicaciones, sistemas, tecnología de la información y redes de datos. Seguidamente se agregaron aquellos títulos vinculados al campo de la comunicación que no llevaban esta palabra en su nombre pero sí alguna de las siguientes: periodismo, relaciones públicas, publicidad, eventos, protocolo, audiovisual, radio, televisión, prensa, internet, redes sociales, cine. Finalmente se excluyeron aquellos títulos que no se encuentran ofertados por las universidades en la edición 2012-2013, verificado esto a través de las web oficiales de las universidades. En total se analizó una muestra compuesta por 135 títulos de Máster universitario (oficiales) de estudios en comunicación y sus diversas ramas y 57 programas de Doctorado.

Mediante un análisis de contenido de las webs oficiales de los programas, se realizó el correspondiente vaciado de información empleando las siguientes variables de estudio: a) designación de la titulación, b) créditos ETCS; c) área temática (audiovisual, comunicación, comunicación digital, comunicación política, comunicación social, intercultural, periodismo, publicidad, relaciones públicas, otros); d) cantidad de plazas ofertadas; e) orientación (investigador, profesional, investigador-profesional); f) año del plan; g) universidad responsable; h) tipo de universidad (pública o privada); i) centro responsable; j) provincia; y k) ciudad.

\section{Desarrollo}

A continuación presentamos los datos arribados del estudio que muestra una clara radiografía de la estructura formativa oficial de titulaciones de Máster universitario y programas de Doctorado en el ámbito de la comunicación de España, ofertados para el curso 2012-2013. Los resultados se encuentran cuantificados en títulos y plazas ofertados (en caso de másteres) a los fines de poder dimensionar las magnitudes de la oferta y el alcance potencial que la misma tienen.

En total nos encontramos con una oferta publicada para el curso 2012-2013 de 135 títulos para 4.742 estudiantes de Máster universitarios, y 57 programas de Doctorado.

${ }^{4}$ https://www.educacion.gob.es/ruct/home 


\subsection{Tipo de Universidad}

En materia de másteres oficiales, el $47 \%$ de la oferta se imparte en universidades privadas (63 titulaciones), siendo el 53\% restante responsabilidad de las universidad públicas, que disponen del $61 \%$ de las plaza totales. De un total de 4.742 plazas, 1.839 están en universidades privadas y el resto, 2.903 candidaturas ofrecen las públicas. El $65 \%$ de los centros que ofertan másteres son universidades públicas (31 de 50 existentes) y el $35 \%$ restantes (17 de 28 existentes) son universidades privadas.

En el caso de los programas de doctorado, de un total de 75 estudios ofertados en $2012 / 2013$, el $25 \%$ corresponden a universidades privadas (14 programas) y el $75 \%$ restantes (43 programas) universidades públicas.

\section{2. Áreas temáticas de los másteres}

La oferta que actualmente se encuentra disponible amplía la tríada que tradicionalmente ha caracterizado los estudios universitarios en comunicación: periodismo, comunicación audiovisual y publicidad y relaciones públicas.

\begin{tabular}{|l|r|r|r|r|}
\hline \multicolumn{1}{|c|}{ Cantidad ofertadas } & \multicolumn{2}{|c|}{ Titulaciones } & \multicolumn{2}{r|}{ Plazas } \\
\hline Relaciones Públicas & 32 & $24 \%$ & 1095 & $23 \%$ \\
\hline Audiovisual & 24 & $18 \%$ & 659 & $14 \%$ \\
\hline Periodismo & 19 & $14 \%$ & 722 & $15 \%$ \\
\hline Comunicación Social & 16 & $12 \%$ & 490 & $10 \%$ \\
\hline Comunicación & 12 & $9 \%$ & 450 & $9 \%$ \\
\hline Publicidad & 10 & $7 \%$ & 285 & $6 \%$ \\
\hline Intercultural & 9 & $7 \%$ & 566 & $12 \%$ \\
\hline Digital & 9 & $7 \%$ & 345 & $7 \%$ \\
\hline Otros & 3 & $2 \%$ & 95 & $2 \%$ \\
\hline Comunicación Politica & 1 & $1 \%$ & 35 & $1 \%$ \\
\hline Total general & 135 & $100 \%$ & 4742 & $100 \%$ \\
\hline \multicolumn{2}{|c|}{ Distribución por área temática en Máster. Fuente: Elaboración propia } \\
\hline
\end{tabular}

En ese sentido, un $21 \%$ de los títulos corresponden al área de comunicación social o comunicación; por su parte, los títulos en comunicación intercultural representan el $7 \%$ de los másteres, junto con aquellos referidos a la comunicación digital.

\subsection{La oferta de posgrado por Universidad}

\begin{tabular}{|c|c|c|}
\hline Cantidad ofertadas & Titulaciones & Plazas \\
\hline Universidad Complutense de Madrid & 8 & 610 \\
\hline Universidad San Pablo-CEU & 15 & 513 \\
\hline Universidad de Alcalá & 2 & 345 \\
\hline Universidad Rey Juan Carlos & 12 & 240 \\
\hline Universidad Ramón Llull & 6 & 210 \\
\hline Universidad Europea de Madrid & 3 & 180 \\
\hline Universidad Autónoma de Barcelona & 7 & 175 \\
\hline IE Universidad & 3 & 130 \\
\hline Universidad de Vigo & 4 & 120 \\
\hline Universidad de Valladolid & 3 & 120 \\
\hline
\end{tabular}




\begin{tabular}{|c|c|c|}
\hline Universidad de Sevilla & 3 & 110 \\
\hline Universidad del Pais Vasco/Euskal Herriko Unibertsitatea & 4 & 107 \\
\hline Universidad Católica San Antonio & 4 & 105 \\
\hline Universidad de Granada & 2 & 100 \\
\hline Universidad Jaume I de Castellón & 1 & 100 \\
\hline Universidad a Distancia de Madrid & 1 & 100 \\
\hline Universidad Internacional de Andalucia y la Universidad de Huelva & 2 & 90 \\
\hline Universidad Pompeu Fabra & 3 & 90 \\
\hline Universidad Cardenal Herrera-CEU & 4 & 90 \\
\hline Universidad Antonio de Nebrija & 6 & 90 \\
\hline Universidad Camilo José Cela & 5 & 75 \\
\hline Universidad de Navarra & 4 & 71 \\
\hline Universidad Carlos III de Madrid & 2 & 65 \\
\hline Universidad de Almeria & 2 & 60 \\
\hline Universitat Internacional Valenciana & 1 & 60 \\
\hline Universidad de Vic & 2 & 60 \\
\hline Universidad de Burgos & 1 & 50 \\
\hline Universitat de València & 1 & 40 \\
\hline Universidad de Girona & 1 & 40 \\
\hline Universidad de Huelva & 1 & 40 \\
\hline Universitat Abat Oliba CEU & 2 & 40 \\
\hline Universidad de Málaga & 1 & 40 \\
\hline Universidad de Málaga y Universidad de Cádiz & 1 & 40 \\
\hline Universidad Miguel Hernández de Elche & 1 & 40 \\
\hline Universidad Autónoma de Madrid & 1 & 36 \\
\hline Universidad de Santiago de Compostela & 1 & 35 \\
\hline Universidad de Alicante & 1 & 35 \\
\hline Universidad Internacional de La Rioja & 2 & 30 \\
\hline Universidad de Deusto & 2 & 30 \\
\hline Universidad Francisco de Vitoria & 2 & 30 \\
\hline Universidad de Zaragoza & 1 & 30 \\
\hline Universidad Rovira i Virgili & 1 & 30 \\
\hline Universidad de La Laguna & 1 & 25 \\
\hline Universidad de Salamanca & 1 & 25 \\
\hline Universidad San Jorge & 1 & 25 \\
\hline Universidad de Oviedo & 1 & 25 \\
\hline Universidad de Córdoba & 1 & 20 \\
\hline Universidad de Barcelona & 1 & 20 \\
\hline Total general & 135 & 4742 \\
\hline
\end{tabular}

Distribución por Universidades Oferta Másteres. Fuente: Elaboración propia

En España, durante el curso 2012/2013, son un total de 48 las universidades que ofertan títulos de Máster oficiales en el ámbito de la comunicación. La Universidad San Pablo-CEU (privada) oferta 15 titulaciones, siendo la Universidad Rey Juan Carlos, la Casa de estudios pública que más títulos oferta, con 12 propuestas.

En el caso de doctorados, los mismos son impartidos por 57 universidades, concentrando un 33\% las universidades Complutense de Madrid (8 programas), Rey Juan Carlos (6 programas) y Autónoma de Barcelona (5 programas). 


\subsection{Distribución territorial según el tipo de Universidad}

Por lo que respecta a la titularidad de la Universidad ofertante, nos encontramos que las públicas ofrecen más títulos de máster (72) que las privadas (63). Empero, esas diferencias son todavía más significativas si consideramos que las universidades públicas son casi el doble que las privadas.

Asimismo, otro aspecto relevante es que la oferta de los centros privados se concentra en Madrid suponiendo el 50\% de la oferta de másteres oficiales de las universidades privadas.

\begin{tabular}{|c|c|c|c|c|}
\hline Cantidad ofertadas & Pública & & Priv & \\
\hline Madrid & 25 & $35 \%$ & 32 & $51 \%$ \\
\hline Barcelona & 11 & $15 \%$ & 10 & $16 \%$ \\
\hline Valencia & 2 & $3 \%$ & 5 & $8 \%$ \\
\hline Segovia & 2 & $3 \%$ & 3 & $5 \%$ \\
\hline Navarra & 0 & $0 \%$ & 4 & $6 \%$ \\
\hline Murcia & 0 & $0 \%$ & 4 & $6 \%$ \\
\hline Bizkaia & 4 & $6 \%$ & 0 & $0 \%$ \\
\hline Pontevedra & 4 & $6 \%$ & 0 & $0 \%$ \\
\hline Sevilla & 4 & $6 \%$ & 0 & $0 \%$ \\
\hline Almeria & 2 & $3 \%$ & 0 & $0 \%$ \\
\hline Gipuzkoa & 0 & $0 \%$ & 2 & $3 \%$ \\
\hline Málaga & 2 & $3 \%$ & 0 & $0 \%$ \\
\hline Granada & 2 & $3 \%$ & 0 & $0 \%$ \\
\hline Zaragoza & 1 & $1 \%$ & 1 & $2 \%$ \\
\hline Huelva & 2 & $3 \%$ & 0 & $0 \%$ \\
\hline La Rioja & 0 & $0 \%$ & 2 & $3 \%$ \\
\hline Burgos & 1 & $1 \%$ & 0 & $0 \%$ \\
\hline Girona & 1 & $1 \%$ & 0 & $0 \%$ \\
\hline Castellón & 1 & $1 \%$ & 0 & $0 \%$ \\
\hline Asturias & 1 & $1 \%$ & 0 & $0 \%$ \\
\hline Tarragona & 1 & $1 \%$ & 0 & $0 \%$ \\
\hline Salamanca & 1 & $1 \%$ & 0 & $0 \%$ \\
\hline Valladolid & 1 & $1 \%$ & 0 & $0 \%$ \\
\hline Santa Cruz De Tenerife & 1 & $1 \%$ & 0 & $0 \%$ \\
\hline Córdoba & 1 & $1 \%$ & 0 & $0 \%$ \\
\hline Alicante & 1 & $1 \%$ & 0 & $0 \%$ \\
\hline A Coruña & 1 & $1 \%$ & 0 & $0 \%$ \\
\hline Total general & 72 & $100 \%$ & 63 & $100 \%$ \\
\hline
\end{tabular}

Si consideramos las provincias donde se concentran los posgrados, el $48 \%$ se encuentra en Madrid, que junto a Barcelona (13\%) suman el 68\% de las plazas de másteres universitarios en comunicación. 


\begin{tabular}{l|r|r|r|r|}
\hline \multicolumn{1}{|c|}{ Cantidad ofertadas } & \multicolumn{2}{|c|}{ Titulaciones } & \multicolumn{2}{r|}{ Plazas } \\
\hline Madrid & 57 & $48 \%$ & 2284 & $42 \%$ \\
\hline Barcelona & 21 & $13 \%$ & 595 & $16 \%$ \\
\hline Segovia & 5 & $5 \%$ & 230 & $4 \%$ \\
\hline Valencia & 7 & $5 \%$ & 230 & $5 \%$ \\
\hline Sevilla & 4 & $3 \%$ & 140 & $3 \%$ \\
\hline Pontevedra & 4 & $3 \%$ & 120 & $3 \%$ \\
\hline Bizkaia & 4 & $2 \%$ & 107 & $3 \%$ \\
\hline Murcia & 4 & $2 \%$ & 105 & $3 \%$ \\
\hline Castellón & 1 & $2 \%$ & 100 & $1 \%$ \\
\hline Granada & 2 & $2 \%$ & 100 & $1 \%$ \\
\hline Huelva & 2 & $2 \%$ & 100 & $1 \%$ \\
\hline Málaga & 2 & $2 \%$ & 80 & $1 \%$ \\
\hline Navarra & 4 & $1 \%$ & 71 & $3 \%$ \\
\hline Almeria & 2 & $1 \%$ & 60 & $1 \%$ \\
\hline Zaragoza & 2 & $1 \%$ & 55 & $1 \%$ \\
\hline Burgos & 1 & $1 \%$ & 50 & $1 \%$ \\
\hline Girona & 1 & $1 \%$ & 40 & $1 \%$ \\
\hline Alicante & 1 & $1 \%$ & 35 & $1 \%$ \\
\hline A Coruña & 1 & $1 \%$ & 35 & $1 \%$ \\
\hline La Rioja & 2 & $1 \%$ & 30 & $1 \%$ \\
\hline Gipuzkoa & 2 & $1 \%$ & 30 & $1 \%$ \\
\hline Tarragona & 1 & $1 \%$ & 30 & $1 \%$ \\
\hline Santa Cruz De Tenerife & 1 & $1 \%$ & 25 & $1 \%$ \\
\hline Salamanca & 1 & $1 \%$ & 25 & $1 \%$ \\
\hline Asturias & 1 & $1 \%$ & 25 & $1 \%$ \\
\hline Córdoba & 1 & $0 \%$ & 20 & $1 \%$ \\
\hline Valladolid & 1 & $0 \%$ & 20 & $1 \%$ \\
\hline Total general & 135 & $100 \%$ & 4742 & $100 \%$ \\
\hline Distribución por Provincias de los Máster. Fuente: Elaboración propia \\
\hline
\end{tabular}

La distribución de programas de Doctorado por provincias también se muestra polarizada. E1 70\% (40 títulos) se concentra en Madrid (24 títulos, de los cuales 18 en Universidades públicas), Barcelona (10 títulos, 7 de los cuales en públicas) y en Valencia (6 títulos).

\subsection{Orientación de las Titulaciones}

El Real Decreto 56/2005 estableció que los másteres debían especializarse en la formación académica o profesional, o bien a promover la iniciación en tareas investigadoras.

\begin{tabular}{|l|r|r|r|r|}
\hline \multicolumn{2}{|c|}{ Cantidad ofertadas } & \multicolumn{2}{|c|}{ Titulaciones } & \multicolumn{2}{c|}{ Plazas } \\
\hline Investigación-Profesional & 44 & $33 \%$ & 1831 & $39 \%$ \\
\hline Profesional & 57 & $42 \%$ & 1721 & $36 \%$ \\
\hline Investigación & 34 & $25 \%$ & 1190 & $25 \%$ \\
\hline Total general & 135 & $100 \%$ & 4742 & $100 \%$ \\
\hline
\end{tabular}

Distribución por Orientación de los Máster. Fuente: Elaboración propia.

En este sentido, los estudios analizados suman un 39\% de las plazas con orientaciones mixtas (Investigación-Profesionales) siendo 44 titulaciones y 1.831 plazas; ofreciendo un $36 \%$ de las plazas (1.721 repartidas en 57 titulaciones) a quienes bus- 
can un perfil profesional en la formación; y 25\% restante repartidas en 34 titulaciones y 1.190 plazas del itinerario investigador.

\section{Conclusiones}

Sin duda, las universidades privadas han irrumpido con fuerza y determinación en la creación de másteres oficiales y doctorados porque no hay que olvidar que este tipo de título debe seguir un proceso lento, farragoso y dilatado de elaboración de la propuesta y de la necesaria aprobación por parte del Ministerio de Educación.

Por término medio, las universidades públicas ofertan en cada Máster una media de 65 plazas, mientras que las universidades privadas presentan una oferta de 30 alumnos por Máster. Eso supone una potencialmente mayor masificación en los másteres ofertados por las universidades públicas.

Respecto a los ámbitos temáticos de los másteres que ofrecen las universidades españolas, nos encontramos el campo de las relaciones públicas como la temática más ofertada, seguida del ámbito audiovisual y del periodismo.

Asimismo, resulta remarcable que existan un gran número de másteres que ofrecen un título con nombre genérico y no especializado, como son los casos de másteres que utilizan la expresión comunicación social o comunicación. Una posible explicación es que son posgrados que se dirigen a titulados universitarios de ámbitos ajenos a la comunicación.

Sobre la orientación de los títulos es reseñable que el mayor número de plazas se oferten con una orientación mixta (investigadora-profesional), seguido de los profesionales y, en último lugar, los de orientación investigadora. Si estructuramos la oferta en los dos grandes bloques, investigador y profesional, y con datos agregados, se ofertan 101 títulos con finalidad profesional frente a los 78 con orientación investigadora.

En este punto resulta muy interesante hacer una reflexión respecto a la oferta de Doctorado. Si consideramos que son 57 programas de Doctorado y 78 los másteres con orientación investigadora, la relación no muestra que no necesariamente encontraremos una correspondencia ni continuidad entre la oferta de segundo y tercer ciclo. En efecto, hay más programas de Máster que funcionan como "período de formación", que programas de Doctorado, que deberían luego complementar el trayecto, haciendo de "período de investigación". Asimismo, si comparamos las plazas ofertadas con orientación investigadora en Máster (3.021 plazas) con los doctorados (57 programas) que luego se supone deberían acoger estos posgraduados, a cada Doctorado le correspondería una media de 53 alumnos. Evidentemente, este hipotético escenario que arroja el análisis de la estructura de la oferta de posgrados en comunicación en España, no es viable y ni sustentable bajo los criterios normativos actuales.

Es notable también como la oferta de posgrado "más rentable" (en relación duración con el coste del crédito) se concentra de forma relativa en las Universidades privadas, mientras que las Universidades públicas cargan luego con la responsabilidad de ofertar los programas doctorales.

En conclusión, podemos ver que la implantación del EEES en el sistema universitario español ha supuesto una verdadera eclosión de postgrados en el ámbito de la comunicación, de modo que la oferta formativa, desde el punto de vista cuantitativo, es 
más que satisfactoria, rica y variada. Más allá del presente trabajo quedaría una indagación de carácter más cualitativo, necesaria para completar el análisis de la situación, recogiendo otra serie de parámetros como la satisfacción del alumnado, los niveles de empleabilidad, índices de calidad, etc., algo que quedará pendiente para subsiguientes investigaciones.

\section{Referencias bibliográficas}

GARCÍA JIMÉNEZ, Antonio y GÓMEZ MOMPART, Josep Lluís (2012a): "Posgrado en Comunicación: una primera aproximación a la situación en España”, en VASSALLO DE LOPES, María Immacolata: Posgrados en Comunicación en Iberoamérica. Políticas Nacionales e Internacionales. San Pablo, ECA-USP., pp. 81-111.

GARCÍA JIMÉNEZ, Antonio y GÓMEZ MOMPART, Josep Lluís (2012b): "Posgrado en Comunicación: una primera aproximación a la situación en España”, en KROHLING KUNSCH, Margarida y MARQUES DE MELO, José: Comunicação Ibero-americana. Sistemas Mediáticos, Diversidade Cultural, Pesquisa e Pós-Graduasao. San Pablo, SOCICOM. CONFIBERCOM, pp. 261-278.

MINISTERIO DE EDUCACIÓN (2010): Datos y cifras del sistema Universitario Español. 2010/2011. En: http://www.educacion.gob.es/educacion/universidades/ [fecha de consulta: 17 de junio de 2012].

OLMEDO SALAR, Silvia; ÁLVAREZ NOBELL, Alejandro; RUIZ MORA, Isabel (2010): "Communication studies in Spain. A process of adaptation to the European Higher Education Area (EHEA)", en SIERRA, Javier: Preparing for the Future: Studies in Communication Sciences in the EHEA. Madrid, Fragua, pp. 39-54.

REAL DECRETO 1393/2007, de 29 de octubre, por el que se establece la ordenación de las enseñanzas universitarias oficiales.

REAL DECRETO 56/2005, de 21 de enero, por el que se regulan los estudios universitarios oficiales de Posgrado.

REAL DECRETO 99/2011, de 28 de enero, por el que se regulan las enseñanzas oficiales de doctorado.

\section{Antonio CASTILLO ESPARCIA}

Universidad de Málaga, Facultad de Comunicación

acastilloe@uma.es

Profesor Titular

\section{Alejandro ÁLVAREZ NOBELL}

Universidad San Jorge (Zaragoza)

aalvarez@usj.es

Personal Docente Investigador

\section{José Antonio MUÑIZ VELÁZQUEZ}

Universidad San Jorge (Zaragoza)

jamuniz@usj.es

Personal Docente Investigador 